\title{
En 2017, le prix Nobel de physique ainsi que la médaille d'or du CNRS récompensent la détection des ondes gravitationnelles
}

\author{
Alain Brillet (Artemis et Virgo) et Thibault Damour (IHES) se sont vu décerner chacun une médaille d'or, \\ tandis que le prix Nobel a été partagé entre trois des principaux initiateurs et responsables du projet \\ LIGO : une moitié a été attribuée à Rainer Weiss (MIT) et l'autre moitié à Barry Barish et Kip Thorne \\ (tous deux de Caltech).
}

Les ondes gravitationnelles (O.G.), dont l'existence est prévue depuis un siècle, avaient été recherchées expérimentalement depuis les années 1960 par des "barres de Weber " (dont une à l'Observatoire de Paris, site de Meudon). Leur découverte était cependant devenue improbable pour la plupart des physiciens dans les années 1970-80, sauf pour de rares pionniers comme R. Weiss aux USA, justement récompensé aujourd'hui. Le résultat positif annoncé par Joseph Weber en 1969 ne fut en effet jamais confirmé, en dépit de nombreuses tentatives.

L'observation d'un premier pulsar binaire, PSR B1913+16, par Russel A. Hulse et Joseph H. Taylor en 1974, a heureusement relancé le débat en établissant vers 1980 l'existence d'un rayonnement gravitationnel comme cause de la diminution au cours du temps de la fréquence orbitale des objets. Le freinage indiquait en effet une perte d'énergie inexplicable autrement. Cette importante déduction leur a valu en 1993 le prix Nobel, et ce n'est pas un hasard si cette même année voit le projet Virgo approuvé par le CNRS et l'année suivante par l'INFN italien. La sensibilité requise n'a cependant été atteinte que récemment, aux USA avec AdvancedLIGO (Laser Interferometer Gravitationalwave Observatory), et en Italie avec Virgo avancé.

Lorsque la première détection directe (événement GW150914, c'est-à-dire Gravitational Waves du 14/09/2015) fut annoncée le 11 février 2016, elle survint donc comme une fantastique nouvelle ! Il s'agissait de la collision de deux trous noirs de l'ordre d'une trentaine de masses solaires chacun ; ceci grâce aux deux détecteurs Advanced-LIGO, l'un situé à Livingstone en Louisiane et l'autre à Hanford dans l'État de Washington. Virgo était en pause à cette date et avait donc " raté " cette première !

L'identification de deux événements moins intenses a rapidement suivi, et les quatrième et cinquième détections ont heureusement été effectuées aussi par Virgo, opérationnel à nouveau depuis le premier août 2017. La triple détection permet en effet une bien meilleure localisation de la source et de pointer tout de suite des télescopes dans la direction indiquée.

Le dernier événement, GW170817, identifié comme la rencontre de deux étoiles à neutrons, a donc été complété par l'observation de rayonnements dans plusieurs domaines : gammas, $\mathrm{X}$, visible et radio. Des raies observées confirment déjà les hypothèses de formation des éléments lourds dans les étoiles à neutrons et ces contreparties électromagnétiques contraignent en outre fortement les théories alternatives à la relativité générale ; les théories à champ scalaire qui prévoient pour les O.G. une vitesse légèrement inférieure à celle de la lumière semblent en particulier devoir être éliminées.

Ces résultats d'une longue recherche ont été récompensés rapidement, par les médailles d'or du CNRS et les prix Nobel 2017. Bien entendu, ces récompenses qui mettent en lumière cinq personnes oublient de très nombreux contributeurs à la construction des interféromètres et à l'analyse des données, indispensables à cette réussite collective impressionnante.

Recruté comme ingénieur CNRS, puis comme chercheur au LHA (Laboratoire de l'Horloge Atomique) puis au LAL (Laboratoire de l'Accélérateur Linéaire) d'Orsay, aujourd'hui chercheur émérite, Alain Brillet est spécialiste des lasers ultrastables, à la fois en fréquence et en puissance, pour

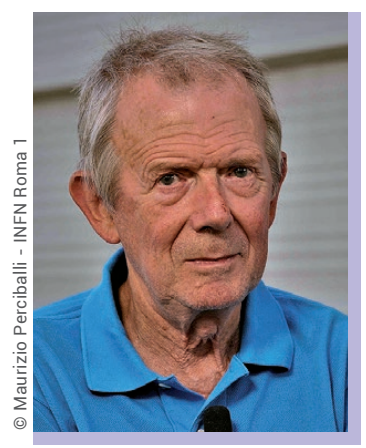

Alain Brillet

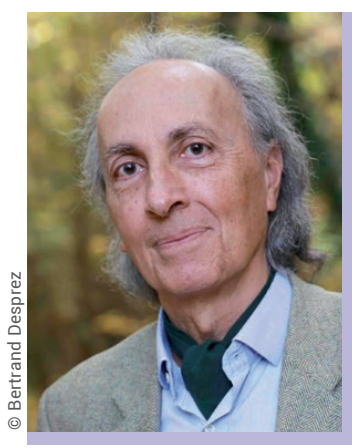

Thibault Damour

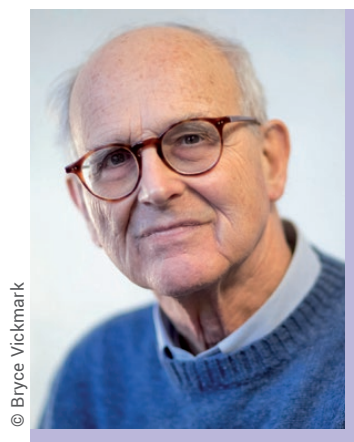

Rainer Weiss

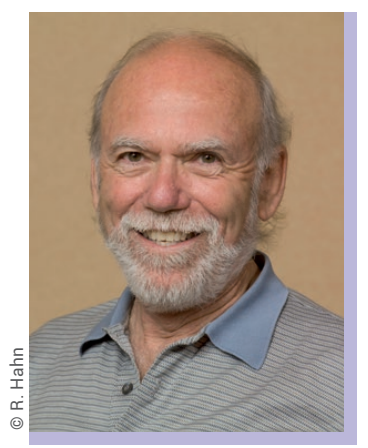

Barry Barish

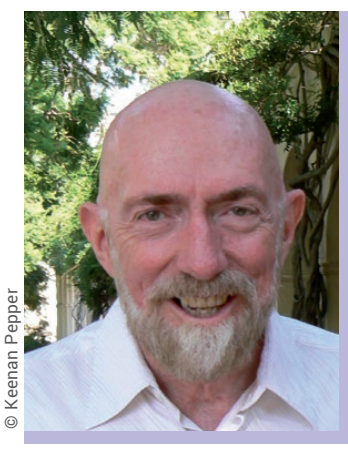

Kip Thorne 

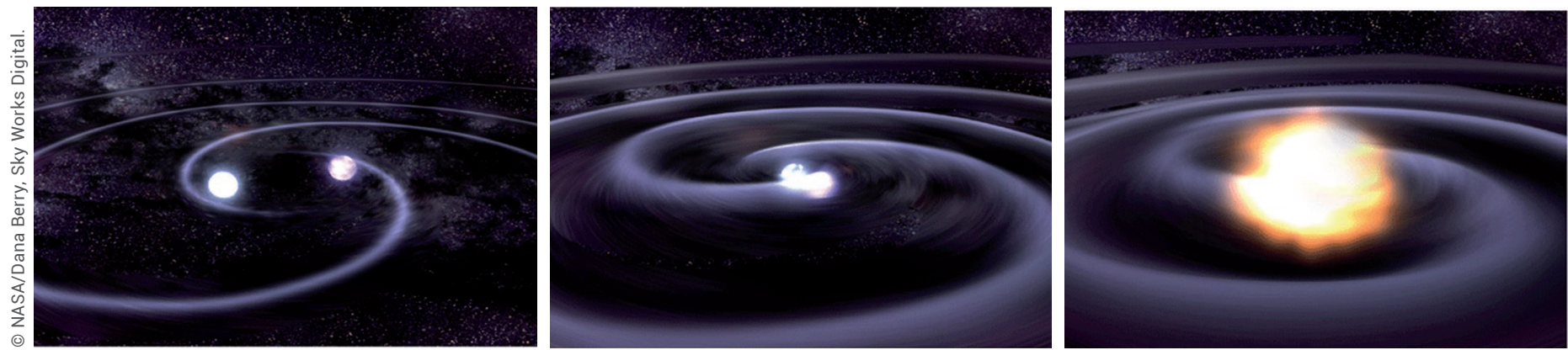

Images de synthèse montrant trois étapes de l'évolution d'une paire de naines blanches, se rapprochant tout en orbitant l'une autour de l'autre, puis entrant en collision. Pendant ce processus, elles émettent de plus en plus d'ondes gravitationnelles. Ce comportement est similaire à la collision de deux étoiles à neutrons.

des applications interférométriques. En 1980, il s'intéresse à leur utilisation possible pour la détection interférométrique des ondes gravitationnelles, avec Jean-Yves Vinet et Catherine Nary Man. En collaboration avec Adalberto Giazotto en Italie, il conçoit le projet d'un détecteur européen de ce type, nommé Virgo parce que l'on espérait des premières sources (de type supernova) dans l'amas de la Vierge. La construction débutera en 1996. Entre 1989 et 2003, Alain Brillet a assuré la direction ou codirection du consortium formé autour du projet Virgo, situé à Cascina près de Pise. Il rejoignit d'ailleurs en 1999 le laboratoire Artemis à l'Observatoire de Nice pour se trouver plus près de Virgo, dont il supervisait la construction. À partir de 2008 il améliora le dispositif en vue du détecteur de deuxième génération Virgo avancé.

Thibault Damour a débuté sa carrière comme chercheur CNRS en 1977 dans le Groupe d'Astrophysique Relativiste de l'Observatoire de Paris-Meudon (GAR devenu ensuite DARC), puis devint en 1989 professeur permanent à l'IHES (Institut des Hautes Études Scientifiques) de Bures-sur-Yvette. Il avait séjourné à Princeton entre 1974 et 1976 pour travailler sur les trous noirs et mit au point à son retour, avec Nathalie Deruelle, la forme de l'émission des ondes gravitationnelles dans le pulsar double récemment détecté. Il a amélioré ainsi la théorie des ondes émises lors de la coalescence supposée de deux objets " compacts ", pour établir le " patron" (modèle d'intensité au cours du temps) du signal qui pourrait être reçu par un détecteur suffisamment sensible. Luc Blanchet, qui avait été son élève, a prolongé depuis avec ses propres méthodes ce travail délicat (voir le $\mathrm{n}^{\circ} \mathbf{5 2}$ de Reflets de la physique pour les articles de Nicolas Arnaud et Luc Blanchet sur la détection des O.G.).

Rainer Weiss, professeur émérite au MIT, est l'inventeur du détecteur interférométrique. En 1967, il demanda à enseigner la relativité générale au MIT et se pencha avec ses étudiants sur le fonctionnement des barres de Weber ; leur résultat n'étant pas confirmé, il revint dans son cours à l'expérience de pensée de deux masses en chute libre équipées de deux horloges échangeant des signaux, pour en évaluer les modifications lors du passage d'une onde gravitationnelle. Il eut alors l'idée de remplacer cet ensemble de deux horloges échangeant des signaux par un interféromètre de Michelson dont les miroirs suspendus seraient les masses en chute libre. Après une étude du bruit dans un tel dispositif, il demanda dès 1972 des subsides pour construire un prototype de $1,5 \mathrm{~m}$. Toujours stimulé par ses étudiants, il s'intéressa au fond diffus cosmologique récemment découvert et se trouva fortement impliqué dans le projet et la réalisation du satellite COBE. Mais ce sont les physiciens du Max Planck Institute qui lui permirent de construire un prototype de $30 \mathrm{~m}$, tandis que Ron Drever faisait de même en Écosse. La rencontre avec Kip Thorne permit ensuite de former un groupe d'étude à Caltech sur la possibilité d'un détecteur d'une sensibilité compatible avec les sources astrophysiques envisageables.

Barry Barish, aujourd'hui professeur émérite à Caltech, a été le directeur de LIGO et le créateur de la collaboration scientifique (de centaines de personnes) associée à l'instrument, déterminante pour l'exploitation des données à laquelle nous assistons aujourd'hui.
Kip Thorne, cofondateur de LIGO avec R. Weiss et R. Drever en 1992, a beaucoup travaillé sur les causes de bruit dans l'instrument et les méthodes d'analyse des données permettant d'identifier un signal. Il fut également à Caltech un théoricien de la relativité générale et a, par exemple, été le premier à faire cours sur les trous noirs en France, en école d'été aux Houches en 1966, après l'avoir fait à Varenne en 1965.

Bien que déjà proposés en 1964 comme explication pour les quasars, par Yakov Zeldovitch et Edwin Salpeter indépendamment, les trous noirs étaient encore à cette date considérés par la plupart des relativistes français (à la suite d'Einstein) comme impossibles.

Le premier pulsar découvert en 1967 par Jocelyne Bell et Anthony Hewish à Cambridge, convainc la communauté de l'existence d'étoiles à neutrons ; ce fait impose la possibilité d'états condensés de la matière et renforce la reconnaissance du trou noir Cygnus X-1, détecté dès 1965, comme invisible perturbateur du mouvement de son étoile compagnon. Les trous noirs sont dès lors repérés comme compagnons invisibles d'objets visibles, stellaires ou galactiques.

Les trous noirs et les étoiles à neutrons sont donc bel et bien aujourd'hui reconnus comme objets de notre science astrophysique, observés plus directement depuis 2015 dans cette nouvelle astronomie des ondes gravitationnelles dont les télescopes sont des interféromètres à lasers.

Christiane Vilain (christiane.vilain@obspm.fr) Laboratoire SPHERE, Université Paris 7 